\title{
Two new species of the primitively segmented spider genus Liphistius Schiödte, 1849 (Mesothelae, Liphistiidae) from Myanmar
}

\author{
Khin Pyae Pyae Aung ${ }^{1,2}$ Xin Xu ${ }^{3,4}$, Wai Wai Lwin', Men Zing Sang', \\ Long $\mathrm{Yu}^{4}, \mathrm{Hao} \mathrm{Liu}^{4}$, Fengxiang Liu ${ }^{4}$, Daiqin $\mathrm{Li}^{5}$
}

I Department of Zoology, University of Yangon, Kamayut Township, Pyay Road, Yangon, 11041, Myanmar 2 Department of Biology, Taungoo Education College, Taungoo, 08101, Myanmar 3 College of Life Sciences, Hunan Normal University, 36 Lushan Road, Changsha 410081, Hunan Province, China 4 State Key Laboratory of Biocatalysis and Enzyme Engineering, and Centre for Behavioural Ecology and Evolution (CBEE), School of Life Sciences, Hubei University, 368 Youyi Road, Wuhan 430062, Hubei Province, China 5 Department of Biological Sciences, National University of Singapore, 14 Science Drive 4, 117543, Singapore

Corresponding author: Xin Xu (xuxin_09@163.com); Daiqin Li (dbslidq@nus.edu.sg)

Academic editor: Jeremy Miller | Received 5 August 2019 | Accepted 27 September 2019 | Published 23 October 2019

http://zoobank.org/70855376-BA7D-4E40-B8C6-6840A6461086

Citation: Aung KPP, Xu X, Lwin WW, Sang MZ, Yu L, Liu H, Liu F, Li D (2019) Two new species of the primitively segmented spider genus Liphistius Schiödte, 1849 (Mesothelae, Liphistiidae) from Myanmar. ZooKeys 882: 29-39. https://doi.org/10.3897/zookeys.882.38811

\begin{abstract}
Two Liphistius species of the primitively segmented spider family Liphistiidae, collected from Loikaw (Kayah State) and Pinlaung (Shan State), Myanmar, are diagnosed and described as new to science based

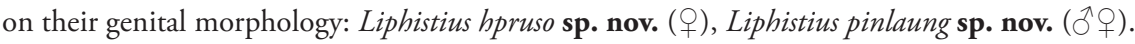

\section{Keywords}

Liphistius, Myanmar, taxonomy, trapdoor spiders

\section{Introduction}

The segmented trapdoor spiders of the family Liphistiidae, the sister lineage to all other extant spiders, are at a pivotal position on the arachnid tree of life (Platnick and Gertsch 1976; Xu et al. 2015a). Liphistiids are often regarded as 'living fossils' 
(Bristowe 1975) since they retain many plesiomorphic characters such as the presence of abdominal tergal plates and the position of the spinnerets on the median area of the opisthosoma (Pocock 1892; Platnick and Gertsch 1976; Haupt 1983, 2003; Coddington and Levi 1991). Two allopatric subfamilies, Liphistiinae Thorell, 1869 and Heptathelinae Kishida, 1923, are distributed in East (China, Japan and Vietnam) and South-east (Laos, Malaysia, Myanmar, Indonesia (Sumatra), and Thailand) Asia, respectively (Xu et al. 2015a, b; World Spider Catalog 2019). Liphistiinae contains 55 described species in the single genus, Liphistius Schiödte, 1849: 33 species from Thailand, 16 from peninsular Malaysia, one from both Thailand and peninsular Malaysia, two from Myanmar, one from Laos, one from Indonesia (Sumatra), and one from both Laos and Thailand (World Spider Catalog 2019). Surprisingly, only two species, L. birmanicus Thorell, 1897 and L. lordae Platnick \& Sedgwick, 1984, have been reported from Myanmar since the first species was described in 1897 (Thorell 1897; Platnick and Sedgwick 1984; Schwendinger 1990; Xu et al. 2015b), given that its landmass is even larger than Thailand, its climate and geological topography are similar to those of Thailand, and it shares the mountain ranges with Thailand across a $10^{\circ}$ latitude range (Fig. 1). Since at least six species in Thailand (L. albipes Schwendinger, 1995, L. bristowei Platnick \& Sedgwick, 1984, L. erawan Schwendinger, 1996, L. jarujini Ono, 1988, L. lahu Schwendinger, 1998, and L. maewongensis Sivayyapram et al., 2017) occur very close to its border with Myanmar, one would expect a comparable species diversity also in Myanmar (Fig. 1).

To document species diversity of Liphistius in Myanmar, we carried out two expeditions in East Myanmar in 2018. In this study, we report two new species of Liphistius after having examined the specimens collected from our expeditions in 2018.

\section{Materials and methods}

\section{Specimen acquisition}

All specimens were collected from Loikaw (Kayah State) and Pinlaung (Shan State), Myanmar (Figs 1,2). They were collected alive and fixed in absolute ethanol if they were adults, and then their right four legs were removed to be stored at $-80{ }^{\circ} \mathrm{C}$ for molecular work. The rest of each specimen was preserved in $80 \%$ ethanol as the voucher for morphological examination.

\section{Morphological examination}

Specimens were examined using an Olympic SZX16 Leica stereomicroscope. Genitalia were cleared in boiling $\mathrm{KOH}$ for a few minutes to dissolve soft tissues, examined and photographed with an Olympic BX53 or SZX7 compound microscope and a Canon $7 \mathrm{D}$ camera. All voucher specimens are deposited at the Centre for Behavioural Ecol- 


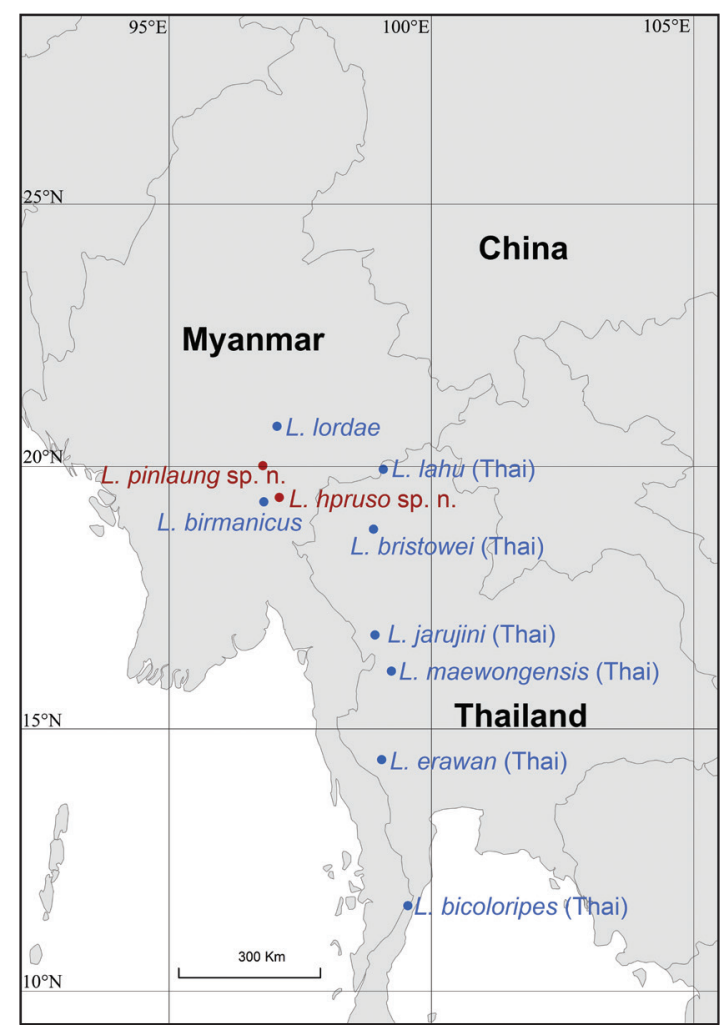

Figure I. A map showing the type localities of ten Liphistius species in Myanmar and Thailand. Two new species are indicated in red solid circles, and two known species in Myanmar and six known species in Thailand are indicated in blue solid circles.

ogy and Evolution (CBEE), College of Life Sciences, Hubei University, Wuhan, Hubei Province, China. Genital anatomical terminology follows Schwendinger and Ono (2011) and Schwendinger (2017). All measurements were carried out under a Leica M205 digital microscope and are given in millimetres. Leg and palp measurements are given in the following order: total leg length (femur + patella + tibia + metatarsus + tarsus), total palp length (femur + patella + tibia + tarsus).

Abbreviations used in the text:

ALE anterior lateral eye;

AME anterior median eye;

CDO central dorsal opening;

CT contrategulum;

E embolus;

GA genital atrium;

PC paracymbium;

PeP paraembolic plate;
PLE posterior lateral eye;

PME posterior median eye;

PPI poreplate;

PS posterior stalk;

RC receptacular cluster;

ST subtegulum;

T tegulum;

TiA tibial apophysis. 

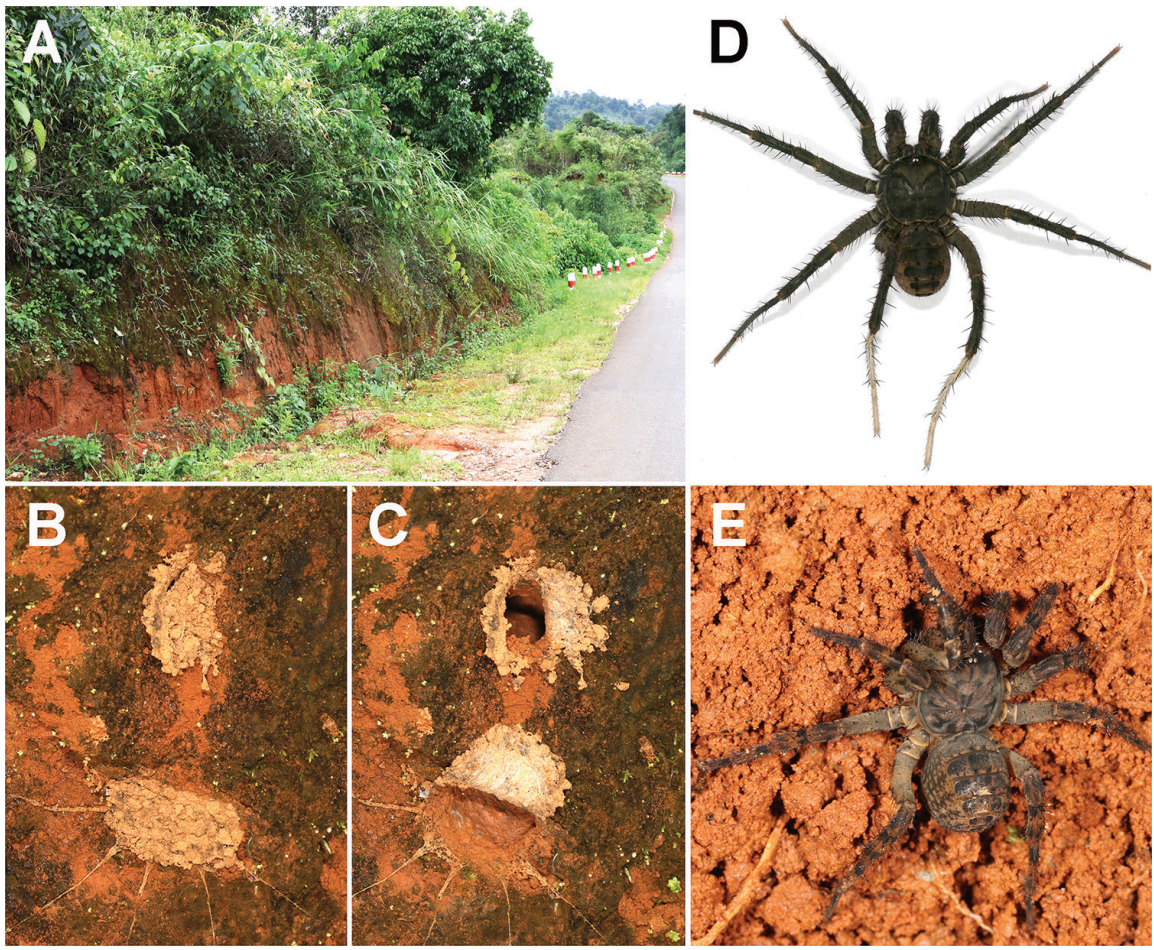

Figure 2. Macrohabitat, burrow with trapdoors, and general somatic morphology (taken in the field) of Liphistius pinlaung sp. nov. A macrohabitat $\mathbf{B}$ a burrow with two trapdoors closed $\mathbf{C}$ a burrow with two trapdoors opened D male (XUX-2018-164, holotype) E female (XUX-2018-162).

\section{Taxonomy}

\section{Family Liphistiidae Thorell, 1869}

Subfamily Liphistiinae Thorell, 1869

Genus Liphistius Schiödte, 1849

Type species. Liphistius desultor Schiödte, 1849

Diagnosis. Liphistius can be distinguished from all other liphistiid genera by the male palp that possesses a tibial apophysis (Fig. 4D, E), and by the presence of a poreplate and a median receptacular cluster in female genitalia (Figs 3B-E, 5A-F).

Distribution. Laos, Malaysia, Myanmar, Indonesia (Sumatra) and Thailand.

\section{Liphistius hpruso sp. nov.}

http://zoobank.org/DC7346A9-F429-4197-A207-7747C24EC9E7

Fig. 3

Type material. Holotype: MYNAMAR . + ; Kayah State, Loi Kaw District, Hpruso, Dokhule, along a small road near Queen of Peace Church; 19.41N, 97.10E; 

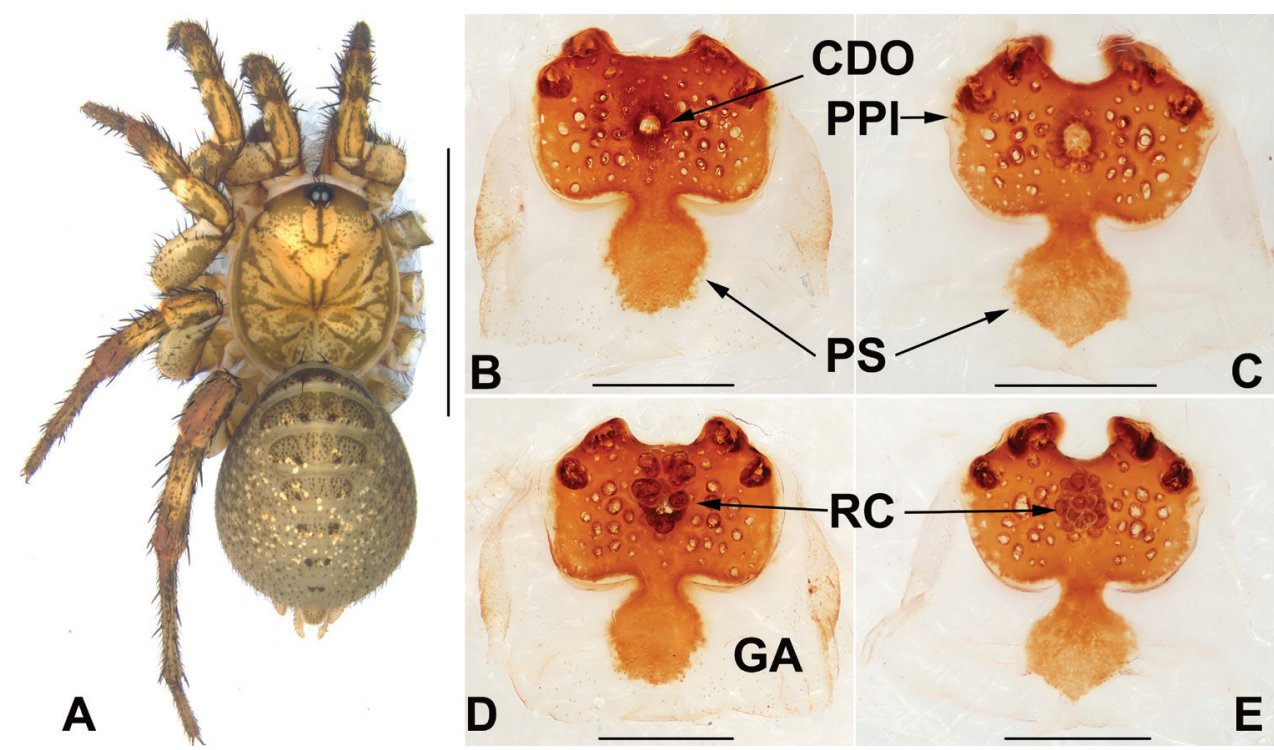

Figure 3. General somatic morphology (taken after fixed by ethanol) and female genitalia of Liphistius hpruso sp. nov. A female (XUX-2018-151, holotype) B, D XUX-2018-151 C, E XUX-2018-152 B, C vulvae, dorsal view D, E vulvae, ventral view. Scale bars: $10 \mathrm{~mm}(\mathbf{A}) ; 0.5 \mathrm{~mm}(\mathbf{B}-\mathbf{E})$.

alt. 1157 m; 17 July 2018; D. Li, F.X. Liu, X. Xu and L. Yu leg.; XUX-2018-151. Deposited in CBEE.

Paratype: MYANMAR $\cdot 1$; same data as for holotype; XUX-2018-152. Deposited in CBEE.

Diagnosis. Females of Liphistius hpruso sp. nov. resemble those of L. birmanicus and $L$. pinlaung sp. nov. by the poreplate with paired anterior lobes and anterolateral lobes, but can be distinguished from those of $L$. birmanicus and L. pinlaung sp. nov. by the globosely receptacular cluster (Fig. 3D, E), and the smaller anterolateral lobes of the pore plate (Fig. 3D, E); from L. pinlaung sp. nov. by the narrower posterior stalk; from the other Liphistius species by the pore plate with similarly sized anterior lobes and anterolateral lobes, and with the narrow posterior stalk (Fig. 3B-E).

Description. Female (holotype). Total length, excluding chelicerae, 16.85. Four thick setae on clypeus (Fig. 3A). Carapace 7.02 long, 6.16 wide, longer than wide, light brown, furnished with few short, scattered bristles. Eight eyes on darkened ocular tubercle, ALE > PLE > PME > AME. Eye sizes and interdistances: AME 0.05, ALE 0.57, PME 0.35, PLE 0.45; AME-AME 0.09, AME-ALE 0.17, PME-PME 0.08, PME-PLE 0.13, ALE-PLE 0.17, ALE-ALE 0.19, PLE-PLE 0.41, AME-PME 0.09. Chelicerae light and glabrous proximally, robust, dark brown; promargin of chelicerae groove with ten denticles of variable size. Labium 0.77 long, 1.47 wide. Sternum 3.61 long, 1.83 wide, brown with several setae. Opisthosoma 9.50 long, 7.53 wide, dark brown, with 12 tergites, and eight spinnerets. Legs brown with strong hairs and spines, long and short black sparse setae, with three tarsal claws. Measurements: palp 10.59 $(3.18+2.20+2.69+2.52), \operatorname{leg}$ I $11.77(3.09+2.31+2.85+1.99+1.52), \operatorname{leg}$ II 12.17 
$(2.72+2.21+2.92+2.49+1.83), \operatorname{leg}$ III $12.45(2.80+2.22+3.16+2.70+1.57)$, leg IV $20.99(4.87+2.79+4.31+5.96+3.06)$.

Female genitalia: vulva with nearly rectangular pore plate; pore plate with similarly sized anterior lobes and anterolateral lobes; distinct transition between the pore plate and posterior stalk (Fig. 3B-E); posterior stalk narrow and long; receptacular cluster spherical and small; central dorsal opening small and circular (Fig. 3B-E).

Male. unknown.

Entomology. "hpruso" refers to the type locality of this species.

Distribution. Myanmar (Loi Kaw District, Kayah State).

\section{Liphistius pinlaung sp. nov.}

http://zoobank.org/1E893A2D-D43C-4B16-A19D-77352D7EE823

Figs 4,5

Type material. Holotype: MYNAMAR · O ; Shan State, Pinlaung Township, ca.14 km to Pinlaung from Pekon; 20.02N, 96.79E; alt. 1410 m; 19 July 2018; D. Li, F.X. Liu, $\mathrm{X} . \mathrm{Xu}$ and L. Yu leg.; XUX-2018-164. Deposited in CBEE.

Paratype: MYNAMAR - 1 万, 5 우; same data as for holotype; XUX-2018-162, 167, 169, 169A, 169B, 169J; 19 July 2018. All specimens deposited in CBEE.

Diagnosis. Males of $L$. pinlaung sp. nov. resemble those of $L$. birmanicus, $L$. lordae and $L$. lahu by the wide paraembolic plate, but can be distinguished from L. birmanicus by the lack of lateral process of paracymbium and by the cumulus with longer and stouter setae (Fig. 4C, D); from L. lordae by the wider tibial apophysis at base (Fig. 4D) and the tegulum with a dentated margin (Fig. $4 \mathrm{C}, \mathrm{F}$ ); from $L$. lahu by the narrower tegulum (Fig. 4C, F) and smaller paracybium (Fig. 4D, E). Females of $L$. pinlaung sp. nov. resemble those of $L$. birmanicus and $L$. hpruso sp. nov. by the poreplate with two pair of lobes, but can be distinguished from L. birmanicus by the wider posterior stalk, and sphere-shaped receptacular cluster (Fig. 5D-F); from L. hpruso sp. nov. by the wider posterior stalk and larger anterior lobes of the poreplate (Fig. 5A-F); from the other Liphistius by the poreplate with four anterior lobes (Fig. 5D-F).

Description. Male (holotype). Total length, excluding chelicerae, 12.71. Carapace 5.86 long and 5.47 wide, longer than wide, olive-green due to being fixed in ethanol immediately after molting, furnished with few short, scattered bristles (Fig. 4A). ALE $>$ PLE $>$ PME $>$ AME, eye sizes and interdistances: AME 0.05, ALE 0.55, PME 0.31, PLE 0.48, AME-AME 0.10, AME-ALE 0.07, PME-PME 0.09, PME-PLE 0.09, ALE-PLE 0.09, ALE-ALE 0.11, PLE-PLE 0.38, AME-PME 0.09. Chelicerae robust, promargin of chelicerae groove with ten strong denticles of variable size. Labium 0.86 long and 0.89 wide, wider than long, fused with sternum and slightly pale olive-green (Fig. 4B). Sternum 2.94 long and 1.05 wide, longer than wide, and a few weakly spined setae on the anterior tip and many long spined setae on the posterior tip, elongated posterior tip (Fig. 4B). Opisthosoma 7.17 long and 4.92 wide, with 12 tergites, the fifth largest, eight spinnerets (Fig. 4B). Legs with strong hairs and spines. Measurements: leg I $16.99(4.32+2.55+3.55+4.66+1.92), \operatorname{leg}$ II $18.06(4.32+2.41+3.74$ 


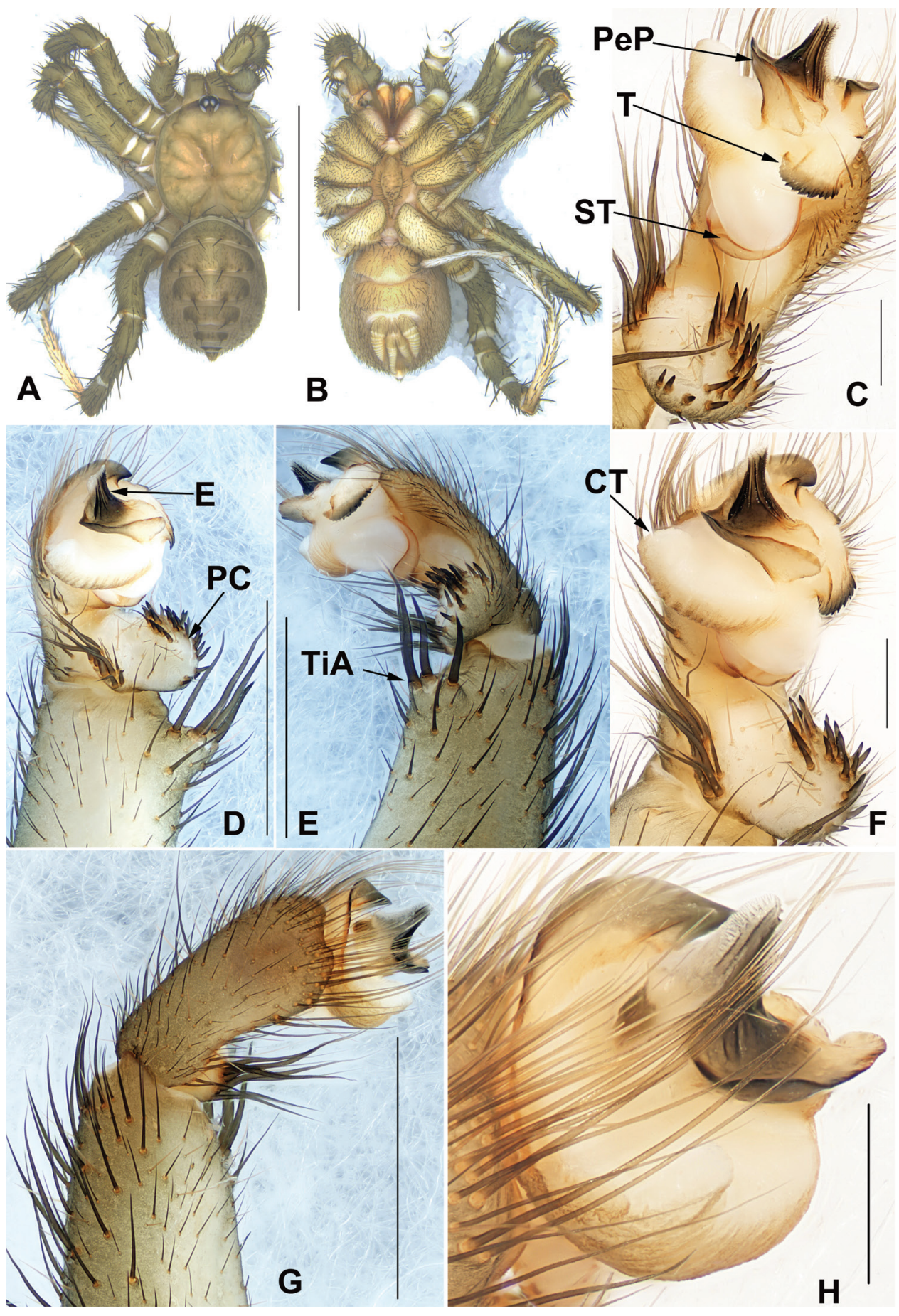

Figure 4. General somatic morphology (taken after fixed by ethanol) and male palp of Liphistius pinlaung sp. nov. (XUX-2018-164, holotype) A, B male: A dorsal view B ventral view C, F, H palp distal view D palp ventral view $\mathbf{E}$ palp retrolateral view $\mathbf{G}$ palp prolateral view. Scale bars: $10 \mathrm{~mm}(\mathbf{A}, \mathbf{B}) ; 2 \mathrm{~mm}$ $(\mathbf{D}, \mathbf{E}, \mathbf{G}) ; 0.5 \mathrm{~mm}(\mathbf{C}, \mathbf{F}, \mathbf{H})$. 


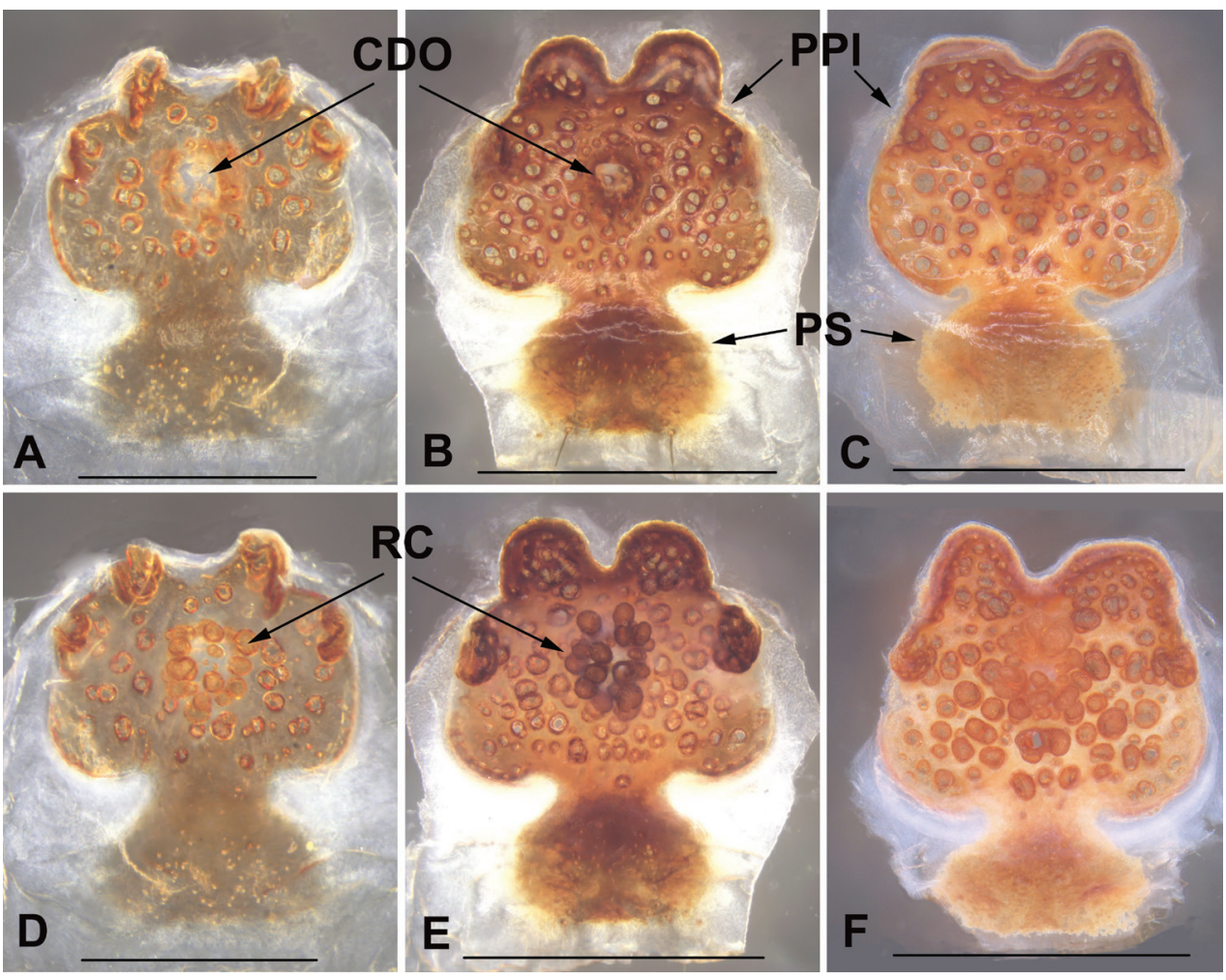

Figure 5. Female genitalia of Liphistius pinlaung sp. nov. A, D XUX-2018-167 B, E XUX-2018-169A C, F XUX-2018-169J A-C vulvae, dorsal view D-F vulvae, ventral view. Scale bars: $0.5 \mathrm{~mm}$ (A, D); $1 \mathrm{~mm}(\mathbf{B}, \mathbf{C}, \mathbf{E}, \mathbf{F})$.

$+5.18+2.41)$, leg III $18.46(4.44+1.85+2.83+6.68+2.66)$, leg IV $20.40(3.56+$ $1.52+4.25+8.46+2.63)$.

Palp: Tibial apophysis with four long spines of different lengths (Fig. 4D, E), paracymbium large and wide, many setae situated at the tip and a row of several tapering spines one the indistinct cumulus (Fig. 4C, D, F); subtegular apophysis weakly developed (Fig. 4C); contrategulum with conical, tip blunt with a short process (Fig. 4C, F), distal edge widely arched, with a smooth and sharp edge (Fig. 4F-H); tegulum small and the terminal apophysis with finely dentated margin (Fig. $4 \mathrm{C}, \mathrm{E}, \mathrm{F}$ ); paraembolic plate short, widely rounded, embolic parts adjacent (Fig. 4D, F, H); embolus long and conical, basally sclerotized, with 3-4 longitudinal ridges that reach to tip (Fig. 4C, D, F).

Female. Total length, excluding chelicerae, 14.46. Carapace 6.70 long, 6.07 wide, light brown, furnished with few short, scattered bristles. Four thick setae on clypeus. Eight eyes on darkened ocular tubercle, ALE > PLE > PME > AME, eye size and interdistances: AME 0.09, ALE 0.61, PME 0.33, PLE 0.47, AME-AME 0.11, AME-ALE 0.16, PME-PME 0.13, PME-PLE 0.13, ALE-PLE 0.14, ALE-ALE 0.14, PLE-PLE 0.43, AME-PME 0.14. Chelicerae proximally glabrous, robust, dark brown; promargin of chelicerae groove with 14 strong denticles of variable size. Labium 0.75 long, 1.19 wide, slightly pale brown. Sternum 3.25 long, 1.59 wide, brown and weakly 


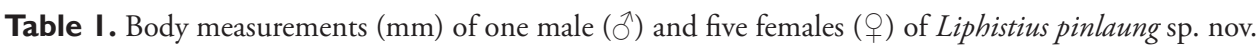

\begin{tabular}{|c|c|c|c|c|c|c|c|c|c|}
\hline \multirow[t]{2}{*}{ Sample number } & \multicolumn{2}{|c|}{ Carapace } & \multicolumn{2}{|c|}{ Opisthosoma } & \multicolumn{2}{|c|}{ Sternum } & \multicolumn{2}{|c|}{ labium } & \multirow{2}{*}{$\begin{array}{l}\text { Body } \\
\text { length }\end{array}$} \\
\hline & length & width & length & width & length & width & length & width & \\
\hline XUX-2018-162 (P) & 6.70 & 6.08 & 8.20 & 5.73 & 3.25 & 1.57 & 0.75 & 1.19 & 14.46 \\
\hline XUX-2018-167 (P) & 5.23 & 4.86 & 4.96 & 3.36 & 2.60 & 1.28 & 0.61 & 0.95 & 10.27 \\
\hline XUX-2018-169 (3) & 6.54 & 6.34 & 7.06 & 5.34 & 2.84 & 1.08 & 0.59 & 0.94 & 13.58 \\
\hline XUX-2018-169A (우) & 7.05 & 5.99 & 7.43 & 5.53 & 3.29 & 1.57 & 0.91 & 1.38 & 14.47 \\
\hline XUX-2018-169B (q) & 7.62 & 6.55 & 7.50 & 5.24 & 3.10 & 1.48 & 0.89 & 1.37 & 14.49 \\
\hline XUX-2018-169J (P) & 7.47 & 6.66 & 7.05 & 4.96 & 3.75 & 1.65 & 0.72 & 1.59 & 14.09 \\
\hline
\end{tabular}

spined, a few setae on the outside of this area, elongated posterior tip. Opisthosoma 8.20 long, 5.73 wide, dark brown, with 12 tergites, the fifth largest, and eight spinnerets. Legs brown with strong hairs and spines, long and short black sparse setae, legs each with three tarsal claws. Measurements: palp $8.59(2.01+1.67+2.65+2.27)$, leg I $11.75(3.39+1.99+3.03+2.01+1.33)$, leg II $12.02(2.69+2.05+3.14+2.45$ $+1.68)$, leg III $13.47(4.19+1.22+3.51+2.49+2.05)$, leg IV $22.4(6.47+2.58+$ $4.38+5.82+3.15)$.

Female genitalia: pore plate with a pair of large anterior lobes and a pair of small, strongly elevated anterolateral lobes, and anterior lobes larger than anterolateral lobes (Fig. 5D-F); distinct transition between the pore plate and posterior stalk (Fig. 5A-F); posterior stalk wide; receptacular cluster spherical and small; central dorsal opening small and circular (Fig. 5A-C).

Entomology. "pinlaung" refers to the type locality of this species.

Distribution. Myanmar (Pinlaung Township, Shan State).

Variation. Body measurements, see Table 1. The examined female genitalia differ from each other; for the specimen of XUX-2018-169A, the central part of anterior and anterolateral lobes of the pore plate are depressed in the dorsal view (Fig. 5B), whereas the depression is absent in the other two specimens (XUX-2018-167 and 169J); the shape and size of anterior and anterolateral lobes of the pore plate, as well as the shape of anterior margin of the pore plate are rather variable (Fig. 5A-F). The size of the receptacular cluster is also slightly different (Fig. 5D-F).

Relationships. Liphistius hpruso sp. nov. and L. pinlaung sp. nov. belong to the birmanicus-group that currently contains $L$. birmanicus, L. lordae and L. lahu based on morphological characters (Schwendinger, 1998). The two new species are closer to $L$. birmanicus than to $L$. lordae and $L$. lahu since their female poreplates possess four anterior lobes (Figs 3B-E; 5D-F).

\section{Acknowledgements}

This study was supported by the grants from the National Natural Sciences Foundation of China (NSFC) (31272324; 31601850), the Hunan Provincial Natural Science Foundation of China (2017JJ3202), the Singapore Ministry of Education AcRF Tier 1 grant (R-154-000-A52-114), ASEAN University Network funding, and Special PhD Program on Zoology from Hubei University. We would like to thank Prof. Thida Lay 
Thwe for her advice and support, and Prof. U Moe Moe Dwe for his help in the field. We are also grateful to Dr Ko Myint for his help for the applications of research permits and spider export permits. In particularly, we would also thank Myanmar Department of Forestry for research permit (NGA/CITES/9/6825/2018) and spider export permit (NWCD/CITES/9/8038/2018) as well as Agri-Food \& Veterinary Authority (AVA) of Singapore for spider import permit. We thank Hirotsugu Ono, Matjaž Kuntner and Rebecca Godwin for their constructive and insightful comments on the manuscript and Shakira Quinones for helping edit the language. We also thank the staff of the Centre for Behavioural Ecology and Evolution (CBEE), Hubei University, College of Life Sciences, Hunan Normal University, and Department of Zoology, University of Yangon for all their help and support throughout this study.

\section{References}

Bristowe WS (1975) A family of living fossil spiders. Endeavour 34: 115-117. https://doi. org/10.1016/0160-9327(75)90130-1

Coddington JA, Levi HW (1991) Systematics and evolution of spiders (Araneae). Annual Review of Ecology and Systematics 22: 565-592. https://doi.org/10.1146/annurev. es.22.110191.003025

Haupt J (1983) Vergleichende Morphologie der Genitalorgane und Phylogenie der liphistiomorphen Webspinnen (Araneae: Mesothelae). I. Revision der bisher bekannten Arten. Zeitschrift für Zoologische Systematik und Evolutionsforschung 21: 275-293. https://doi. org/10.1111/j.1439-0469.1983.tb00296.x

Haupt J (2003) The Mesothelae - monograph of an exceptional group of spiders (Araneae: Mesothelae) (Morphology, behaviour, ecology, taxonomy, distribution and phylogeny). Zoologica 154: 1-102.

Ono H (1988) Liphistiid spiders (Araneae, Mesothelae) of northwest Thailand. Bulletin of the National Museum of Nature and Science Tokyo (A) 14: 35-41.

Platnick NI, Gertsch WJ (1976) The suborders of spiders: a cladistic analysis (Arachnida, Araneae). American Museum Novitates 2607: 1-15.

Platnick NI, Sedgwick WC (1984) A revision of the spider genus Liphistius (Araneae, Mesothelae). American Museum Novitates 2781: 1-31.

Pocock RI (1892) XXXVIII. - Liphistius and its bearing upon the classification of spiders. Journal of Natural History Series 6(10): 306-314. https://doi.org/10.1080/00222939208677416

Schwendinger PJ (1990) On the spider genus Liphistius (Araneae: Mesothelae) in Thailand and Burma. Zoologica Scripta 19: 331- 351. https://doi.org/10.1111/j.1463-6409.1990. tb00262.x

Schwendinger PJ (1995) New Liphistius species (Araneae, Mesothelae) from southern Thailand and northern Malaysia. Zoologica Scripta 24(2): 143-156. https://doi. org/10.1111/j.1463-6409.1995.tb00396.x

Schwendinger PJ (1996) New Liphistius species (Araneae, Mesothelae) from western and eastern Thailand. Zoologica Scripta 25(2): 123-141. https://doi.org/10.1111/j.1463-6409.1996. tb00155.x 
Schwendinger PJ (1998) Five new Liphistius species (Araneae, Mesothelae) from Thailand. Zoologica Scripta 27: 17-30. https://doi.org/10.1111/j.1463-6409.1998.tb00426.x

Schwendinger PJ (2017) A revision of the trapdoor spider genus Liphistius (Mesothelae: Liphistiidae) in peninsular Malaysia; part 1. Revue Suisse de Zoologie 124: 391-445. https:// doi.org/10.5281/zenodo.893555

Schwendinger PJ, Ono H (2011) On two Heptathela species from southern Vietnam, with a discussion of copulatory organs and systematics of the Liphistiidae (Araneae: Mesothelae). Revue Suisse de Zoologie 118: 599-637. https://doi.org/10.5962/bhl.part.117818

Sivayyapram V, Smith DR, Weingdow S, Warrit N (2017) A new Liphistius species (Mesothelae: Liphistiidae: Liphistiinae) from Thailand, with notes on its natural history. Journal of Arachnology 45(3): 287-295. https://doi.org/10.1636/JoA-S-17-028.1

Thorell T (1897) Viaggio di Leonardo Fea in Birmania e regionivicine. LXXIII. Secondo saggio sui Ragnibirmani. I. Parallelodontes. Tubitelariae. Annali del Museo Civico di Storia Naturale di Genova 37: 161-267.

World Spider Catalog (2019) World Spider Catalog, Version 20.5. Natural History Museum Bern. http://wsc.nmbe.ch [access on 10 September 2019]

Xu X, Liu FX, Cheng R-C, Chen J, Xu X, Zhang ZS, Ono H, Pham DS, Norma-Rashid Y, Arnedo MA, Kuntner M, Li D (2015a) Extant primitively segmented spiders have recently diversified from an ancient lineage. Proceedings of the Royal Society B: Biological Sciences 282: 20142486. https://doi.org/10.1098/rspb.2014.2486

Xu X, Liu FX, Chen J, Ono H, Li D, Kuntner M (2015b) A genus level taxonomic review of primitively segmented spiders (Mesothelae, Liphistiidae). ZooKeys 488: 121-151. https:// doi.org/10.3897/zookeys.488.8726 